\title{
Alerts for managing postpartum haemorrhage
}

\author{
S Fawcus, MA (Oxon), MB BCh, FRCOG \\ Department of Obstetrics and Gynaecology, Faculty of Health Sciences, University of Cape Town, South Africa
}

Corresponding author: S Fawcus (sue.fawcus@uct.ac.za)

\begin{abstract}
A review of maternal deaths from postpartum haemorrhage (PPH) in 2014 - 2016 raises concern at the slow rate of reduction of mortality. Folder review showed that $87.8 \%$ of deaths were avoidable and identified 'red flags': common omissions (poor problem recognition), commissions (incorrect treatment) and areas of substandard care (inadequate monitoring and/or management). The lessons learnt have led to key practice points for managing PPH, which are presented in this article. These include: referral to an appropriate level of care for delivery, medications for prevention of $\mathrm{PPH}$, and how facilities can be prepared for PPH to detect it timeously - before the onset of coagulopathy. Emergency management of severe obstetric haemorrhage includes resuscitation, identifying the cause/s of the bleeding, medical treatment of $\mathrm{PPH}$, non-medical interventions for $\mathrm{PPH}$ in the labour ward, early recourse to theatre with ongoing bleeding and a description of effective surgical interventions. The problems associated with referral of patients with PPH are discussed. Updated management algorithms for PPH in South Africa are presented.
\end{abstract}

S Afr Med J 2018;108(12):1013-1017. DOI:10.7196/SAMJ.2018.v108i12.13420

\section{Latest data on deaths from obstetric haemorrhage in South Africa}

There is ongoing concern at the slow rate of reduction of mortality from obstetric haemorrhage $(\mathrm{OH})$ in South Africa (SA), despite several educational interventions. $\mathrm{OH}$ was the third most common cause of maternal death for the triennium 2014 - 2016, accounting for 624 deaths or $16.9 \%$ of the total, ${ }^{[1]}$ compared with $684(15.8 \%)$ deaths in 2011 - 2013. The OH-related maternal mortality ratio (MMR) was 22.7 deaths per 100000 live births, i.e. $<24.3$ in $2011-2013$, but $>18.8$ in 2005 - 2007. There was considerable provincial variation in the MMR; haemorrhage was highest in Limpopo Province (33.5), Mpumalanga Province (32.7) and North West Province (28.9) and lowest in Western Cape Province (7.4).

The major causes of death from haemorrhage were bleeding associated with caesarean delivery (CD) (34.9\%), uterine rupture (15.1\%), abruptio placentae (12.9\%), uterine atony (9.5\%) and retained placenta (6.9\%). Of concern was the persisting high number of deaths due to bleeding after $\mathrm{CD}$, which was described in an interim publication. ${ }^{[2]}$ The majority of $\mathrm{OH}$ deaths $(93.9 \%)$ occurred at public hospitals, $32.4 \%$ at district hospitals, $30.4 \%$ at regional hospitals and $27.3 \%$ at tertiary hospitals. This might not reflect the level of care where delivery occurred, as many women were referred from district level, with bleeding after delivery or $\mathrm{CD}$, to a regional or tertiary facility. ${ }^{[2]}$ Assessors judged the majority (87.8\%) of $\mathrm{OH}$ deaths to be possibly or probably avoidable. Review of the folders of $\mathrm{OH}$ deaths identified 'red flags', e.g. common omissions (poor problem recognition), commissions (incorrect treatment) and areas of substandard care (inadequate monitoring and/or management). These lessons learnt have led to the following key practice points for managing $\mathrm{OH}$.

\section{Referral to an appropriate level of care for delivery}

Although it is not possible to predict with certainty which women will have postpartum haemorrhage (PPH), there are some known antenatal and intrapartum predictive factors. ${ }^{[3]}$ Antenatally, these include anaemia, multi-fibroid uterus, previous $\mathrm{PPH}$, previous $\mathrm{CD}$, placenta praevia, pre-eclampsia, parity $\geq 5$, multiple pregnancies and antepartum haemorrhage. Intrapartum factors include prolonged first stage of labour, prolonged second stage of labour, chorioamnionitis and abruptio placentae. Such women need to be identified for delivery at district or regional hospital level, where skilled doctors of the appropriate level and blood products are available. When an identified woman lives far from the referral hospital, maternity waiting areas can enable her to be closer to the appropriate facility when her labour commences.

\section{Prevention of postpartum haemorihage}

Active management of the third stage of labour with 10 IU intramuscular oxytocin reduces the risk of PPH by $60 \%$ and should be employed routinely for birth at a facility. ${ }^{[4]}$ This can be followed by a 20 IU oxytocin infusion or intramuscular oxytocin/ergometrine combination preparation for women at increased risk of PPH. High blood pressure (BP) and cardiac disease are contraindications to ergometrine administration. Similarly, at $\mathrm{CD}$, prophylaxis with oxytocin should be employed. Because of concern regarding hypotension from rapid intravenous oxytocin boluses, especially in combination with spinal anaesthesia, smaller slower boluses should be given, supplemented with an infusion. ${ }^{[5]}$ Alternatively, oxytocin or the oxytocin/ergometrine combination can be administered intramuscularly if there are no contraindications ${ }^{[6]}$ Other interventions that reduce the likelihood of PPH include vigilant intrapartum monitoring to prevent obstructed labour by timely $\mathrm{CD}$, availability of skill for operative vaginal delivery to avoid unnecessary $\mathrm{CD}$ in the second stage of labour, and good surgical technique for CD.

\section{Being prepared}

Good ward-level management by a senior nurse, with support of the facility procurement department, is important to ensure continuous availability of essential medications, fluids, infusion sets and equipment for balloon tamponade. If all of the above are in a 'PPH box', time will not be wasted searching for items during an emergency, and the completeness of the items can be more easily 
checked on a daily basis. Furthermore, it is very important that there is an adequate supply of blood products. This could be an on-site blood bank (as at tertiary hospitals and some regional hospitals). However, such a resource is not available for district hospitals and the majority of regional hospitals, where there must be efficient oversight to ensure a functioning fridge with adequate supplies of emergency blood, which is replaced in an ongoing manner after use. Also, fresh dried plasma (FDP) should be procured as a pharmacy item and kept in the labour ward and/or theatre. Regular on-site emergency drills (emergency obstetric scenario training), where the team practise how to take care of a severe $\mathrm{PPH}$, are essential to ensure that they know what to do and how to do it. ${ }^{[7]}$

\section{Early detection of haemorrhage (before coagulopathy)}

After massive blood loss ( $>2 \mathrm{~L}$ ), a coagulopathy develops and all attempts to stop the bleeding become more complex and hazardous. Therefore, early recognition of PPH with prompt treatment can prevent progression to severe shock and coagulopathy. The mandatory 2 hours of monitoring after vaginal delivery with 15-minute observations of vital signs should be adhered to. A similar period of monitoring after $\mathrm{CD}$ in a designated recovery area should also be a standard routine. The African Surgical Outcomes Study (ASOS) ${ }^{[8]}$ identified CD as the most common surgical operation performed in Africa and identified the immediate postoperative period as a critical time for early detection of surgical complications. Colour-coded earlywarning monitoring charts aid in the identification of problems. ${ }^{[9]}$

During this monitoring period - beware the 'slow trickle, which may add up to severe blood loss and lead to sudden decompensation; and beware the 'tachycardia', which is usually the first sign of above-average blood loss and should not be ignored.

Not all $\mathrm{OH}$ is visible: beware the concealed bleeding from abruptio placentae, ruptured uterus, paravaginal haematoma, or post-CD intra-abdominal bleeding. A patient with a retained placenta does not remain stable, and severe PPH is likely to ensue if the placenta is not removed at the site where she delivered. Therefore, midwives at primary level need to be trained to perform manual removal of the placenta. Early resuscitation with crystalloid fluids is essential to replace blood volume, but after $3 \mathrm{~L}$ of crystalloid alone, there is an increased risk of coagulopathy. Therefore, blood usually needs to be given before this stage during ongoing bleeding. ${ }^{[10]}$
A shock index (pulse/systolic BP) of $>0.9$ is an early sign of hypovolaemia. It is also an indication to call a doctor and for intervention. ${ }^{[11]}$ Early involvement of the doctor as part of the team to manage $\mathrm{PPH}$ is essential. Importantly, doctors should not wait for the BP to drop before attending to the patient, because by this time she is likely to have bled at least $2 \mathrm{~L}$.

\section{Emergency management of severe obstetric haemorrhage}

After 1.5 - $2 \mathrm{~L}$ of blood loss, there will be symptoms and signs of shock (dizziness, confusion, low BP, tachycardia, pallor and oliguria). Severe $\mathrm{PPH}$, if untreated, can lead to death within 2 3 hours (NB: referral is not treatment).
Beware if the pulse is twice as high as the systolic BP reading. A shock index of $>1.7$ requires an urgent response and should initiate calling the $\mathrm{OH}$ team, where such teams exist, or the most senior medical and nursing staff on site, in the absence of such teams, as in smaller hospitals. ${ }^{[11]}$ This is a dire emergency; the patient must not be left until bleeding stops and signs improve, and help must be summoned immediately. In regional and tertiary hospitals, haematologists, the blood bank and in some situations, interventional radiologists need to be alerted. In other regional and all district hospitals the most senior doctor, including a doctor experienced in anaesthesia, needs to be summoned. Aggressive resuscitation and obstetric management to establish the cause

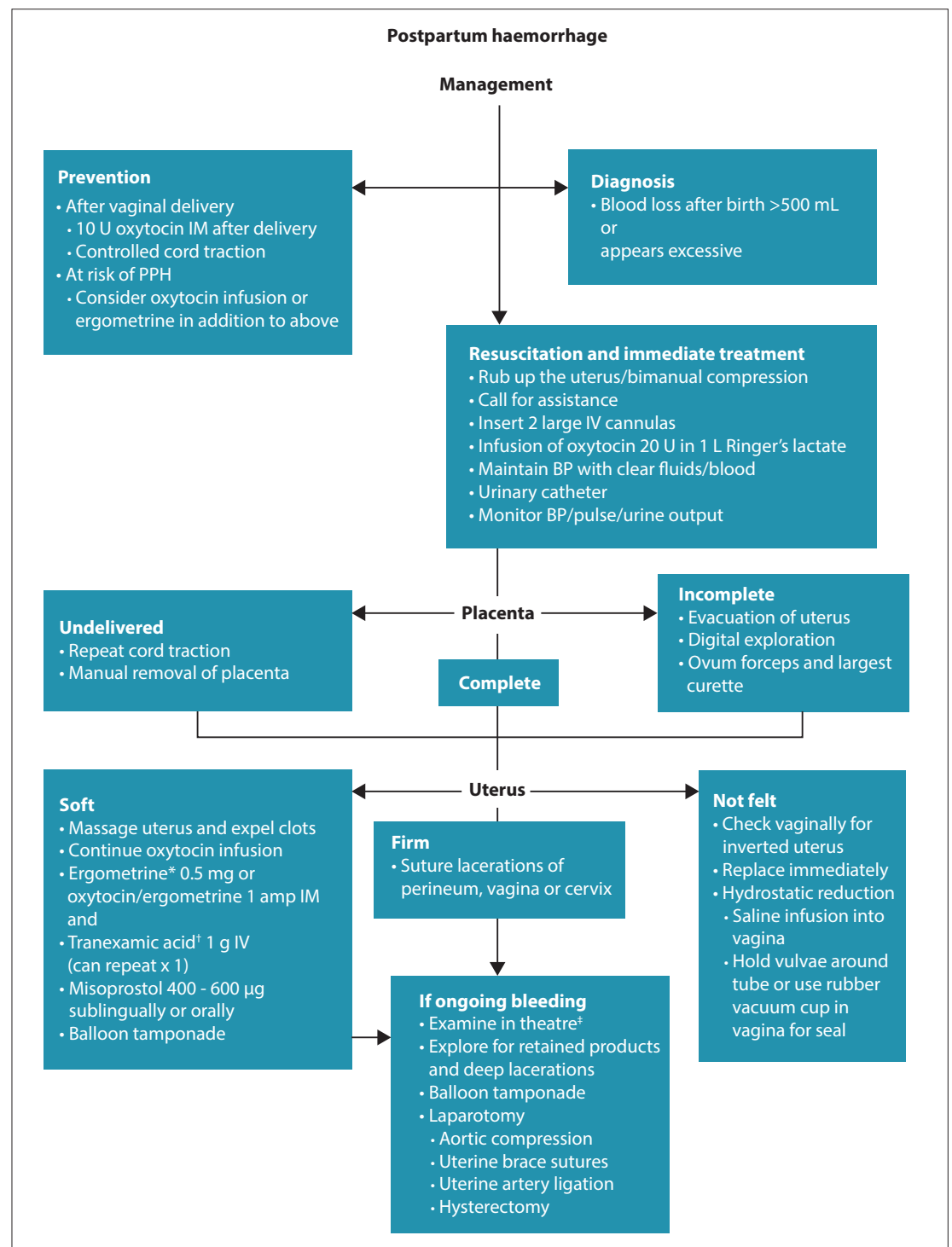

Fig. 1. Postpartum haemorrhage after vaginal delivery. $(I M=$ intramuscular; $P P H=$ postpartum haemorrhage; $I V=$ intravenous; $B P=$ blood pressure; amp = ampoule. $)\left({ }^{*}\right.$ Avoid if increased $B P$ or cardiac condition; ${ }^{\dagger}$ Avoid if venous thromboembolism or artificial heart valves; ${ }^{*}$ In facilities with no theatre, patient will need emergency referral. To optimise condition, consider: balloon catheter tamponade; non-pneumatic anti-shock garment, if available.) 
of the bleeding and stop it, must take place concurrently, with another person available to document findings and treatment and make phone calls.

\section{Resuscitation}

Use of blood products, such as red cells, fresh frozen plasma (FFP), cryoprecipitate and platelets, can be goal-directed in wellresourced settings, where immediate haematology results can guide blood-product component therapy replacement. ${ }^{[10]}$ There is a move to the earlier administration of fibrinogen-rich products. ${ }^{[10-12]}$ Unfortunately, in SA, this is not the norm and bloodproduct replacement is 'formula driven. ${ }^{\text {' }}{ }^{13,14]}$ The national PPH guideline recommends the following: after $2 \mathrm{~L}$ of crystalloid, +/- colloid, transfuse $2 \mathrm{U}$ of red cells, followed by $1 \mathrm{U}$ of FDP or FFP for every further red cell unit given. The use of point-of-care tests, such as HemoCue (HemoCue, SA) for haemoglobin estimation, can assist in decision-making, and in the future, thrombo-elastography may have value in poor-resource settings, although this is still being researched. ${ }^{[12]}$

\section{Identify cause/s of bleeding}

This step is often overlooked, with PPH being seen as a diagnosis in itself requiring only medical therapy. While uterine atony is the most common cause of PPH after vaginal delivery, and it is recommended to always commence an oxytocin infusion as the first line of treatment, it is also important to consider other causes. Overlooking major vaginal trauma or even ruptured uterus can cause delays in definitive treatment and result in death. Also, there may be more than one cause of $\mathrm{PPH}$, e.g. retained products or a cervical tear might co-exist with uterine atony.

\section{Medical treatment of postpartum haemorrhage}

This includes uterotonic drugs to contract the uterus, which are given sequentially; followed 10 - 15 minutes later by an oxytocin infusion if there is no response after oxytocin/ergometrine or ergometrine (if no contraindications), as well as tranexamic acid. The last, a fibrinolytic inhibitor, was not previously in the SA PPH protocol, but the WOMAN trial ${ }^{[15]}$ indicated that it reduces PPH mortality compared with placebo when administered in addition to standard therapy. A dedicated World Health Organization guideline ${ }^{[16]}$ specifies the importance of using it early in the $\mathrm{PPH}$ algorithmic approach, as its effect is reduced if given after 3 hours of onset of $\mathrm{PPH}$. It is now included in the new SA $\mathrm{PPH}$ protocol. Contraindications include artificial heart valves and current or previous thromboembolic disease. Prostaglandins have also been used for PPH. Prostaglandin $\mathrm{F}_{2}$-alpha is no longer available globally. There is a place for sublingual misoprostol to promote uterine contraction, but avoid high doses, which lead to morbidity. ${ }^{[17]}$ It could be a second-line agent in patients with contraindications to oxytocin/ergometrine or when there is cold-chain failure for storing the other uterotonic drugs that require refrigeration.

\section{Non-medical interventions for postpartum haemorrhage in the labour ward}

These interventions, including manual removal of the placenta, can be done in community health clinics, midwife obstetric units (MOUs) and district hospitals. They include bimanual compression, aortic compression, suture of perineal tears and balloon tamponade. The last can involve purpose-made devices, such as the Ellavi balloon (Sinapi, SA), Rusch catheter (Teleflex Medical, USA) or Bakri balloon (Cook Medical, USA), or 'home-made' devices that use condoms or surgical gloves to make the balloon. ${ }^{[18]}$ Urgent referral is essential in facilities that do not have on-site doctors or theatre facilities, such as clinics/MOUs, and in patients in whom the initial treatment measures described above do not succeed. Advice must be obtained from the referral hospital before transfer.

\section{Early recourse to theatre}

If bleeding persists despite all medical and nonmedical labour ward interventions, another cause needs to be sought. Early recourse to theatre is needed in this situation, and also when immediate post-delivery findings suggest

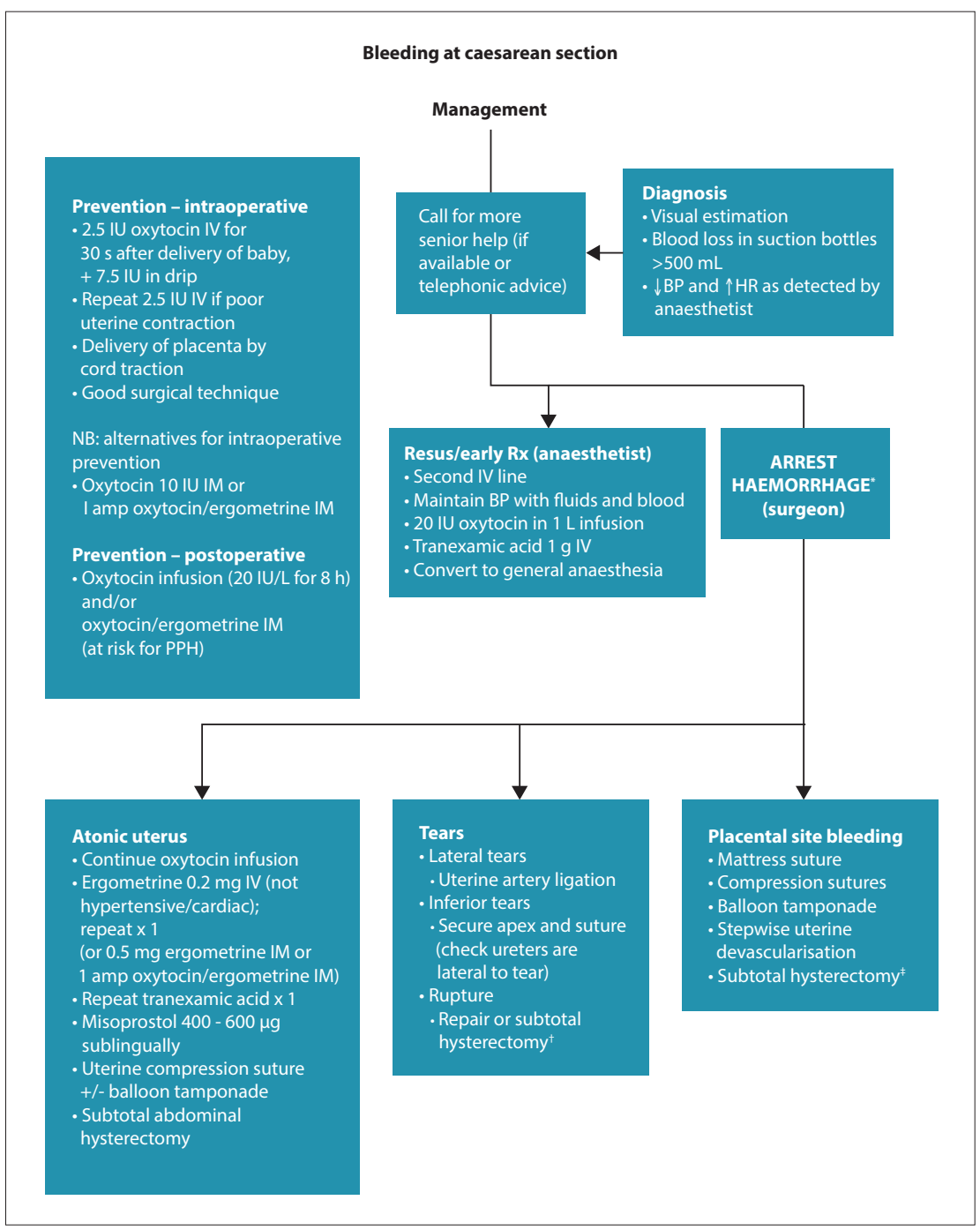

Fig. 2. Excessive bleeding at caesarean delivery. (IV = intravenous; $I M=$ intramuscular; amp = ampoule; $P P H=$ postpartum haemorrhage; $B P=$ blood pressure; $H R=$ heart rate; Resus = resuscitation; $R x=$ treatment. $)\left({ }^{*}\right.$ Proceed immediately to subtotal abdominal hysterectomy (STAH) if: uterine rupture (irreparable); placenta percreta; 'If lack of skill for STAH, apply uterine tourniquet and refer; "Nonpneumatic anti-shock garment, if available.) 
retained placenta, retained products or trauma not amenable to repair in the labour ward, e.g. deep vaginal tears or suspected cervical/ uterine trauma. Consent for examination under anaesthesia and additional procedures that may be required, such as laparotomy or hysterectomy, needs to be obtained at this stage. Examination under anaesthesia can be done at district hospital level and is preferable to transferring unstable patients without some form of definitive or temporising treatment. Early recourse to theatre for patients with bleeding after $\mathrm{CD}$ is very important and can also be done at a district hospital after informing the referral hospital about the patient.

\section{Surgical interventions for postpartum haemorrhage}

Removal of placental products, suture of vaginal/cervical tears, balloon tamponade, repair of uterine tears at $\mathrm{CD}$, and laparotomy for uterine compression sutures can be performed at all levels of hospital. Details for these procedures are not described in this article, but can be referred to in the Monograph on the Management of Postpartum Haemorrhage ${ }^{[13]}$ and the caesarean section monograph, ${ }^{[19]}$ and are taught in the essential steps in managing obstetric emergencies (ESMOE) modules. ${ }^{[7]}$ Hysterectomy is a life-saving procedure that must be done when the abovementioned conservative surgical measures fail, or immediately in cases of extensive uterine rupture or placenta increta/ percreta. However, it is a specialised operation requiring greater gynaecological skill than CD. The surgical skill is often not available at district hospitals. Ideally, attempts should be made to train doctors at district hospital level to perform a subtotal hysterectomy, but this may not be feasible; eligible cases would be infrequent, and there is often a high turnover of doctors. Alternatively, the doctor must perform conservative surgical measures and apply a uterine tourniquet before packing, closing and referring to a tertiary hospital. ${ }^{[2]}$

\section{Management algorithms for postpartum haemorrhage}

These have been revised in response to recent research, new technologies, review of influential PPH guidelines, new guidance on resuscitation in haemorrhagic shock and findings from the Saving Mothers reports (Figs 1 - 3)

\section{Referral of patients with ongoing obstetric haemorrhage}

While the emphasis is on resuscitation and all possible interventions at the site where the delivery and $\mathrm{PPH}$ occurs, there will always be

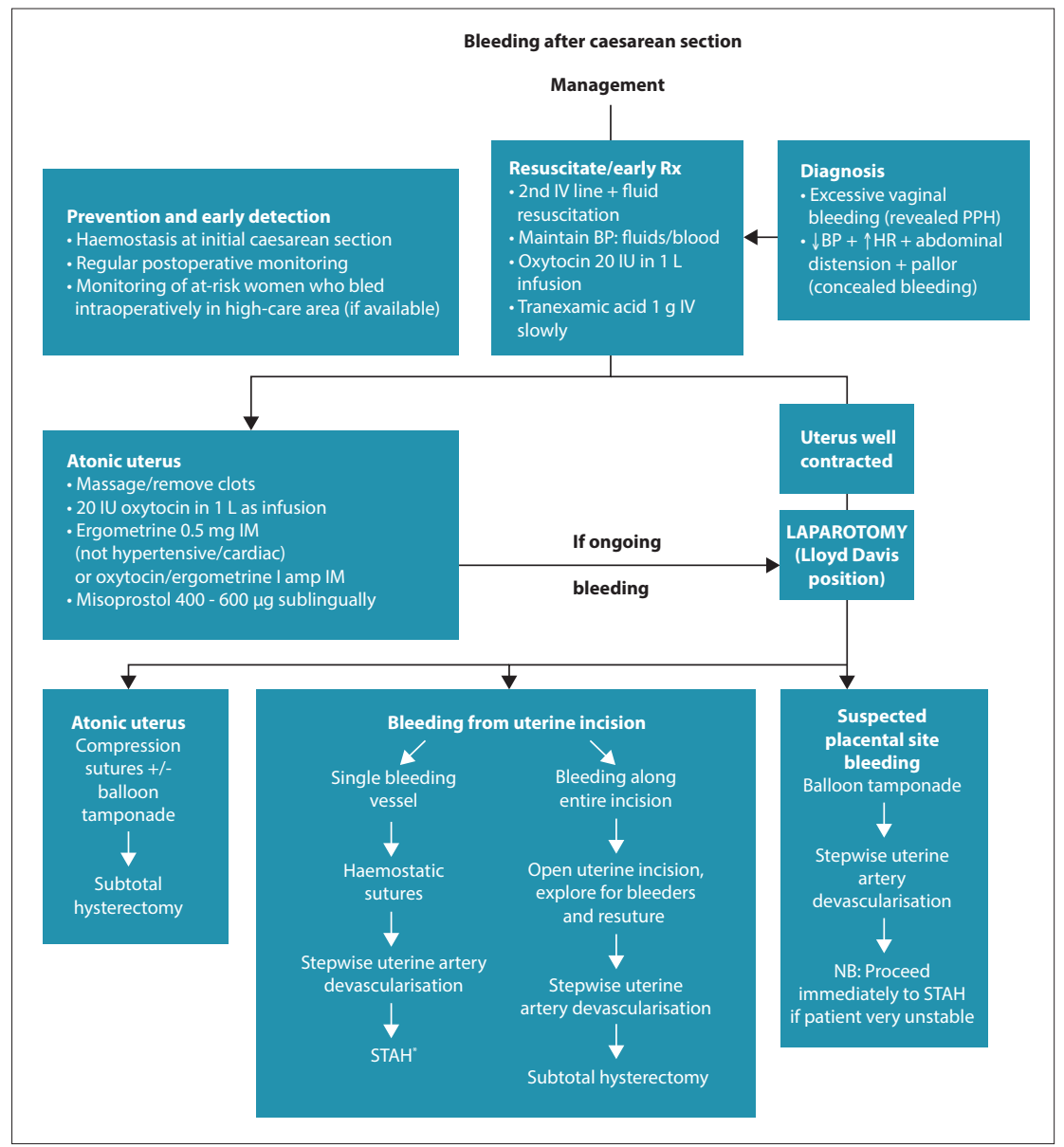

Fig. 3. Excessive bleeding after caesarean delivery. $(R x=$ treatment; $I V=$ intravenous; $B P=$ blood pressure; $P P H=$ postpartum haemorrhage; $H R=$ heart rate; $I M=$ intramuscular; amp = ampoule; STAH = subtotal abdominal hysterectomy.) (*If lack of skill for STAH, apply uterine tourniquet and refer; non-pneumatic anti-shock garment, if available.)

a need to refer patients, after PPH, to a higher level of care. This is because of the structure of the maternity system in SA, with different levels of care and surgical skill. In some patients the bleeding would have been stopped prior to transfer, which is required for management in an intensive care unit and blood replacement at a regional or tertiary hospital. In other patients, the bleeding would not have been definitively stopped and no more could be done at the referring institution. Unfortunately, many deaths occur in such patients during transit or shortly after arrival at the next level of care, which may be compounded by ambulance delays. To avoid this, the following is recommended: good communication with the receiving hospital the senior obstetrician must advise the referring doctor of all possible temporising interventions before transfer; resuscitation and treatment to continue up to transfer, which should not be discontinued after transfer arrangements have been made; availability of paramedicassisted ambulances to be improved in most provinces so that resuscitation and treatment can be continued during transfer, and if possible a staff member must accompany the patient to assist with this. The non-pneumatic antishock garment is a neoprene garment with compression panels on the lower limbs and abdomen, which treats shock by maintaining blood flow to essential organs during transit. ${ }^{[21]}$ It is soon to be procured and piloted in SA and has great potential for improving the condition of shocked women during transfer so that they arrive alive and can be definitively managed at the next level of care.

Declaration. None.

Acknowledgements. National Committee for Confidential Enquiries into Maternal Deaths.

Author contributions. Sole author.

Funding. None.

Conflicts of interest. None.

1. National Department of Health. Saving Mothers 2014 - 2016. The Seventh Report of the National Committee for Confidential Enquiries into Maternal Deaths in South Africa. Pretoria: $\mathrm{NDoH}, 2017$.

2. Fawcus S, Pattinson RB, Moodley J, et al. Maternal deaths from bleeding associated with caesarean delivery: A national emergency. S Afr Med J 2016;106(5):472-476. https://doi.org/10.7196/ SAMJ.2016.v.106i5.10821 
3. Mavrides E, Allard S, Chandraharan E, et al., on behalf of the Royal College of Obstetricians and Gynaecologists. Prevention and management of postpartum haemorrhage. Br J Obstet Gynaecol 2016;124:e106-e149. https://doi.org/10.1111/1471-0528.14178

4. Tuncalp O, Souza JP, Gulmezoglu M. New recommendations for the prevention and treatment of

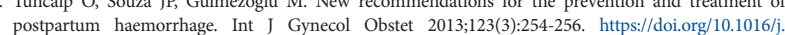
postpartum haen

5. Dyer R, van Dyk D, Dresner A. The use of uterotonics during caesarean section. Int J Obstet Anaesth 2010;19(3):313-319. https://doi.org/10.1016/j.ijoa.2010.04.011

6. Farina Z, Fawcus S. Oxytocin: Ensuring appropriate use and balancing efficacy with safety. S Afr Med J 2015;105(4):271-274. https://doi.org/10.7196/samj.917

. National Committee on Confidential Enquiries into Maternal Deaths. Saving Mothers. Essential Steps in the Management of Common Conditions Associated with Maternal Mortality. Pretoria: National Department of Health, 2007.

8. Biccard BM, Madiba TE, Kluyts HL, et al., and ASOS investigators. Perioperative patient outcomes in the African Surgical Outcomes Study: A 7-day prospective observational cohort study. Lancet 2018;391(10130):1589-1598. https://doi.org/10.1016/S0140-6736(18)30001-1

9. Smith C, Brown J. Could pregnant Canadian women benefit from an early obstetric warning system? J Obstet Gynaecol Can 2018;40(4):397-398. https://doi.org/10.1016/j.jogc.2017.101012

10. Cannon J. Hemorrrhagic shock. N Engl J Med 2018;378:370-379. https://doi.org/10.1056/NEJMra1705649

11. Nathan HL, Ayadi AE, Hezelgrave NL, et al. Shock index: An effective predictor of outcome in postpartum haemorrhage? Br J Obstet Gynaecol 2015;122(2):268-276. https://doi.org/10.1111/14710528.13206

12. Pavord S, Maybury H. How I treat postpartum haemorrhage. Blood 2015;125(18):2759-2769. https:// doi.org/10.1182/blood-2014-10-512608

13. National Committee on Confidential Enquiries into Maternal Deaths. Monograph on the Management of Postpartum Haemorrhage. Pretoria: National Department of Health, 2010.
14. National Department Health. Guidelines for Maternal Care in South Africa: A Manual for Clinics, Community Health Centres and District Hospitals. Pretoria, NDoH, 2015.

15. WOMAN Trial Collaborators. Effect of early tranexamic acid administration on mortality hysterectomy, and other morbidities in women with post-partum haemorrhage (WOMAN): An
(WOM international, randomised, double-blind, placebo controlled trial. Lancet 2017;389(10084):2105-2116 https://doi.org/10.1016/S0140-6736(17)30638-4

16. World Health Organization. Recommendation on Tranexamic Acid for the Treatment of Postpartun Haemorrhage. Geneva: WHO, 2017

17. Hofmeyr GJ, Gulmezoglu M, Novikova N, Lawrie T. Postpartum misoprostol for preventing maternal mortality and morbidity. Cochrane Database Syst Rev 2013;(7):CD008982 https://doi. org/10.1002/14651858.CD008982.pub2

18. Tindell $\mathrm{K}$, Garfinkel R, Abu-Haydon E, et al. Uterine balloon tamponade for the treatment of postpartum haemorrhage in resource poor settings: A systematic review. Br J Obstet Gynaecol 2012;120(1):5-14. https://doi.org/10.1111/j.1471-0528.2012.03454

19. National Committee on Confidential Enquiries into Maternal Deaths. Caesarean Section Monograph Pretoria: National Department of Health, 2013

20. Wessels $\mathrm{U}$, Mayat $\mathrm{N}$. Use of a uterine tourniquet as a temporizing measure during transfer of patien with obstetric haemorrhage. O\&G Forum 2013;23(3):29-31

21. Pileggi-Castro C, Nogueira-Pileggi V, Tuncalp O, Oladapo O, Vogel JP, Souza JP. Non-pneumatic anti-shock garment for improving maternal survival following severe postpartum haemorrhage: A systematic review. Reprod Health 2015;12:28. https://doi.org/10.11861s2978-015-0012-0

Accepted 24 May 2018 